\begin{tabular}{|c|l|}
\hline Title & $\begin{array}{l}\text { Molecular dynamics study of kinetic boundary condition at an interface between a poly atomic vapor and its condensed } \\
\text { phase }\end{array}$ \\
\hline Author(s) & Ishiyama, Tatsuya; Y ano, Takeru; Fujikawa, Shigeo \\
\hline Citation & $\begin{array}{l}\text { PHY SICS OF FLUIDS, 16(12), 4713.4726 } \\
\text { https://doi.org/L0.1063/1811674 }\end{array}$ \\
\hline Issue Date & 2004 \\
\hline Doc URL & http://hdl.handle.net/2115/5841 \\
\hline Rights & Copyright $\odot 2004$ A merican Institute of Physics \\
\hline Type & article \\
\hline File Information & PF16-12.pdf \\
\hline
\end{tabular}

Instructions for use 


\title{
Molecular dynamics study of kinetic boundary condition at an interface between a polyatomic vapor and its condensed phase
}

\author{
Tatsuya Ishiyama, Takeru Yano, ${ }^{\text {a) }}$ and Shigeo Fujikawa \\ Division of Mechanical Science, Graduate School of Engineering, Hokkaido University, \\ Sapporo 060-8628, Japan
}

(Received 21 May 2004; accepted 7 September 2004; published online 11 November 2004)

\begin{abstract}
The kinetic boundary condition for the Boltzmann equation at an interface between a polyatomic vapor and its liquid phase is investigated by the numerical method of molecular dynamics, with particular emphasis on the functional form of the evaporation part of the boundary condition, including the evaporation coefficient. The present study is an extension of a previous one for argon [Ishiyama, Yano, and Fujikawa, Phys. Fluids 16, 2899 (2004)] to water and methanol, typical examples of polyatomic molecules. As in the previous study, molecular dynamics simulations of vapor-liquid equilibrium states and those of evaporation from liquid into a virtual vacuum are carried out for water and methanol. In spite of the formation of molecular clusters in the vapor phase and the presence of the preferential orientation of molecules at the interface, essentially the same results as in the previous study are obtained. When the bulk liquid temperature is relatively low, the evaporation part is the product of the half range Maxwellian for the translational velocity of molecules of saturated vapor at the temperature of the bulk liquid phase, the equilibrium distribution of rotational energy of molecules at the temperature, and the evaporation coefficient (or the condensation coefficient in the equilibrium state). The evaporation coefficients of water and methanol are determined without any ambiguity as decreasing functions of the temperature, and are found to approach unity with the decrease of the temperature. (C) 2004 American Institute of Physics. [DOI: 10.1063/1.1811674]
\end{abstract}

\section{INTRODUCTION}

The behavior of a vapor adjacent to an interface between the vapor and its condensed phase can be determined by solving the Boltzmann equation (or a model equation) with a kinetic boundary condition at the interface. ${ }^{1,2}$ The kinetic boundary condition widely used can be written as the sum of an evaporation part $\alpha f^{\mathrm{e}}$ and a reflection part $(1-\alpha) f^{\mathrm{r}}$,

$$
f^{\text {out }}=\alpha f^{\mathrm{e}}+(1-\alpha) f^{\mathrm{r}} \quad\left(\xi_{z}>0\right)
$$

where $f^{\text {out }}$ is the distribution function of outgoing molecules from the interface, $\alpha$ is a parameter between zero and unity, sometimes called the condensation coefficient, $f^{\mathrm{e}}$ is the equilibrium distribution of saturated vapor at the temperature of the condensed phase, $f^{\mathrm{r}}$ is usually the distribution function of the diffuse reflection at the temperature, and $\xi_{z}$ is the velocity component normal to the interface (for simplicity, we consider an interface at rest). A number of problems have been solved with this type of kinetic boundary condition (see Refs. 1 and 2 and references therein). However, its physical validity still remains to be established, and the related studies on the basis of the molecular dynamics (MD) method have just been started. ${ }^{3-6}$ In the present paper, we investigate the validity of this type of kinetic boundary condition at the interface of a polyatomic vapor and its own liquid phase by

\footnotetext{
a) Author to whom correspondence should be addressed. Telephone: +81-11706-6430. Fax: +81-11-706-7889. Electronic mail:

yano@mech-me.eng.hokudai.ac.jp
}

MD method, with particular emphasis on the functional form of the evaporation part.

Previously, we have studied the kinetic boundary condition at an interface between argon vapor and its condensed phase. ${ }^{6}$ In the study, we have executed MD simulations of vapor-liquid (or solid) equilibrium states and those of evaporation from the liquid (or solid) phase into a virtual vacuum. Thereby, we have demonstrated that, in the case that the temperature of the bulk condensed phase $T_{\ell}$ is near the triple point temperature of argon, the distribution function for molecules evaporating into virtual vacuum is given by $\alpha_{e} \rho_{v} \hat{f}^{*}$, where $\rho_{v}$ is the saturated vapor density, $\rho_{v} \hat{f}^{*}$ is the halfMaxwellian of saturated vapor defined as

$$
\rho_{v} \hat{f}^{*}=\frac{\rho_{v}}{\left(2 \pi R T_{\ell}\right)^{3 / 2}} \exp \left(-\frac{\xi_{x}^{2}+\xi_{y}^{2}+\xi_{z}^{2}}{2 R T_{\ell}}\right) \quad\left(\xi_{z}>0\right)
$$

( $R$ is the gas constant and $\xi_{x}$ and $\xi_{y}$ are the velocity components tangential to the interface), and $\alpha_{e}$ is the evaporation coefficient (or the condensation coefficient in the equilibrium state) defined by the ratio of a mass flux evaporating into virtual vacuum $\left\langle J_{\mathrm{evap}}^{\mathrm{sp}}\right\rangle$ to the outgoing mass flux in the equilibrium state $\left\langle J_{\text {out }}\right\rangle_{\mathrm{e}}$,

$$
\alpha_{e}=\frac{\left\langle J_{\mathrm{evap}}^{\mathrm{sp}}\right\rangle}{\left\langle J_{\mathrm{out}}\right\rangle_{\mathrm{e}}}
$$

(see Fig. 1 and Sec. II for detail). The evaporation part in the kinetic boundary condition represents spontaneous evaporation, which is independent of the condition of vapor, and 


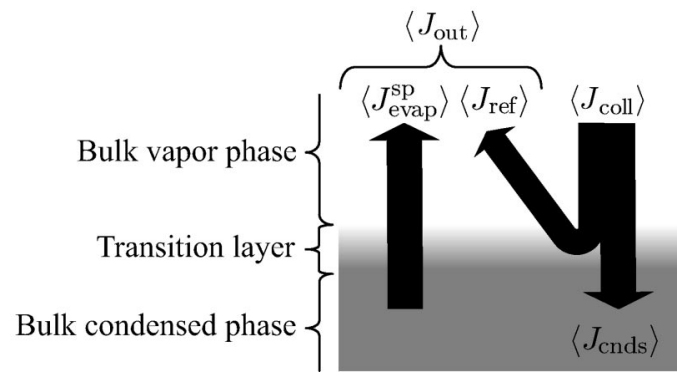

FIG. 1. Molecular fluxes at the interface.

hence it is unchanged whether the condensed phase is contact with the vapor or exposed to a virtual vacuum. Therefore, one can regard the distribution function for molecules evaporating into virtual vacuum, Eq. (2), as the evaporation part in the kinetic boundary condition. We have thus shown that the evaporation part in Eq. (1) is valid in relatively low temperature cases. ${ }^{6}$ The determination of the functional form of the reflection part in various nonequilibrium conditions may require a number of nonequilibrium MD simulations because it depends on the state of the vapor and such nonequilibrium computations are underway.

Here, we note that there is an experimental result corresponding to the virtual vacuum simulation, ${ }^{7}$ where it is shown that the velocity distribution of sodium atoms emitted from liquid surface is a Maxwellian and the evaporation coefficient is unity. The evaporation of dimer sodium has also been studied there. However, the distributions of angular velocity and rotational energy of the dimer have not been discussed, and the evaporation coefficient has not been measured.

In this paper, we shall extend our previous study to the cases of water and methanol, typical examples of polyatomic molecules for which the vapor-liquid coexistence is possible at room temperature. The simulations are carried out in a similar way to the previous one, but a more careful and detailed analysis is required, because of the additional degrees of freedom for internal motions and the polarity due to uneven sharing of the bonding electron pair. In particular, the latter induces the formation of molecular clusters in vapor phase and the presence of the preferential orientation of molecules at the interface. Nevertheless, we arrive at essentially the same conclusions as the previous study. That is, the evaporation part can be expressed as

$$
\alpha_{e} \rho_{v} \hat{f}^{*} \hat{g}^{*} \quad\left(\xi_{z}>0\right),
$$

in relatively low temperature cases, where $\rho_{v} \hat{f}^{*}$ is the halfMaxwellian for translational motions of center of mass of a polyatomic molecule, which is equal to that given in the right-hand side of Eq. (2) for a monatomic molecule, and $\hat{g}^{*}$ is the equilibrium distribution associated with internal motions of polyatomic molecule of $n$ degree of freedom,

$$
\hat{g}^{*}=\frac{E^{n / 2-1}}{\Gamma(n / 2)\left(k T_{\ell}\right)^{n / 2}} \exp \left(-\frac{E}{k T_{\ell}}\right) \quad(0 \leqslant E<\infty)
$$

( $k$ is the Boltzmann constant, $E$ is the energy of internal motion of one molecule, and $\Gamma$ is the gamma function). The symbol ^ ${ }^{\wedge}$ ignifies a normalized distribution function and the superscript $*$ represents that the distribution of molecules is in an equilibrium state. Equation (4) has been utilized for the analysis of the effects of internal degree of freedom on inverted temperature profiles between evaporating and condensing parallel plates. ${ }^{8}$ The main objective of the present study is to examine the validity of Eq. (4) with Eqs. (2), (3), and (5) for the cases of water and methanol by the numerical method of molecular dynamics.

In the following section, we shall provide the outline of the method of analysis. After the description of the computational method in Sec. III, the numerical results and discussion are given in Sec. IV, and followed by conclusions in Sec. V.

\section{OUTLINE OF ANALYSIS}

As in the previous study, ${ }^{6}$ we first define a distribution function of spontaneous evaporation $f_{\text {evap }}^{\text {sp }}$ as a distribution function of molecules evaporating from the interface and independent of the condition of incident vapor molecules. This means that $f_{\text {evap }}^{\mathrm{sp}}$ is unchanged whether the condensed phase is contact with the vapor or exposed to virtual vacuum, and it should be determined by the temperature in the bulk condensed phase only. An arbitrary $f^{\text {out }}$ given by MD simulation can then be split into two parts, $f_{\mathrm{evap}}^{\mathrm{sp}}$ and the remainder, i.e.,

$$
f^{\text {out }}=f_{\text {evap }}^{\text {sp }}+f^{\text {ref }}\left(\xi_{z}>0\right),
$$

where $f^{\text {ref }}=f^{\text {out }}-f_{\text {evap. }}^{\text {sp }}$ Putting $f^{\text {out }}$ in the form of Eq. (6) enables us to verify the evaporation part of Eq. (1) in the method explained below. The splitting may also be regarded as the extraction of an inherent property of the bulk condensed phase from $f^{\text {out }}$. We shall remark that we do not intend classifying every individual outgoing molecule as either evaporated or reflected one in a vapor-liquid two-phase system; the complete classification may be impossible as mentioned in Refs. 5 and 6.

If the condensed phase is exposed to virtual vacuum, there are no incoming and reflected molecules at the interface, and hence we have

$$
f^{\text {out }}=f_{\text {evap }}^{\text {sp }}\left(\xi_{z}>0\right) .
$$

Accordingly, the functional form of $f_{\text {evap }}^{\text {sp }}$ can be determined by an MD simulation of evaporation into virtual vacuum. In Sec. IV, we execute the virtual vacuum simulation by eliminating vapor molecules at a distance near the interface, and evaluate a normalized distribution function $\rho_{c}^{-1} f_{\text {evap }}^{\text {sp }}$ by counting the number of evaporating molecules, where $\rho_{c}$ is the density of molecules evaporating into virtual vacuum,

$$
\rho_{c}=\iiint_{\xi_{z}>0} \int_{0}^{\infty} f_{\mathrm{evap}}^{\mathrm{sp}} d E d \xi_{x} d \xi_{y} d \xi_{z} .
$$

The examination of the functional form of $\rho_{c}^{-1} f_{\mathrm{evap}}^{\mathrm{sp}}$ in Sec. IV D shows that

$$
\rho_{c}^{-1} f_{\mathrm{evap}}^{\mathrm{sp}}=2 \hat{f}^{*} \hat{g}^{*} \quad\left(\xi_{z}>0\right)
$$

holds in relatively low temperature cases for water and methanol, where $\hat{f}^{*}$ and $\hat{g}^{*}$ are defined by Eqs. (2) and (5), 
respectively. From Eqs. (2), (5), and (9), we have

$\left\langle J_{\mathrm{evap}}^{\mathrm{sp}}\right\rangle=\iiint_{\xi_{z}>0} \int_{0}^{\infty} \xi_{z} f_{\mathrm{evap}}^{\mathrm{sp}} d E d \xi_{x} d \xi_{y} d \xi_{z}=\rho_{c} \sqrt{\frac{2 R T_{\ell}}{\pi}}$,

$\left\langle J_{\text {out }}\right\rangle_{\mathrm{e}}=\iiint_{\xi_{z}>0} \int_{0}^{\infty} \xi_{z} \rho_{v} \hat{f}^{*} \hat{g}^{*} d E d \xi_{x} d \xi_{y} d \xi_{z}=\rho_{v} \sqrt{\frac{R T_{\ell}}{2 \pi}}$.

Substituting Eqs. (10) and (11) into Eq. (3) immediately gives

$$
\rho_{c}=\frac{1}{2} \alpha_{e} \rho_{v} .
$$

Thus, we can show that $f_{\mathrm{evap}}^{\mathrm{sp}}=\alpha_{e} \rho_{v} \hat{f}^{*} \hat{g}^{*}$ for water and methanol.

The parameter $\alpha$ in the right-hand side of Eq. (1) has often been called the condensation coefficient. The precise definition of the condensation coefficient $\alpha_{c}$ is

$$
\alpha_{c}=\frac{\left\langle J_{\text {cnds }}\right\rangle}{\left\langle J_{\text {coll }}\right\rangle},
$$

where $\left\langle J_{\text {cnds }}\right\rangle$ is a condensing mass flux at the interface and $\left\langle J_{\text {coll }}\right\rangle$ is a collision mass flux. ${ }^{9}$ By definition, $\alpha_{c}$ is affected by the condition of vapor, while $\alpha_{e}$ is uniquely determined by the temperature of the condensed phase [see Eqs. (3), (10), and (11)]. In the equilibrium state, as can readily be seen from Fig. 1, $\left\langle J_{\text {coll }}\right\rangle=\left\langle J_{\text {out }}\right\rangle=\left\langle J_{\text {out }}\right\rangle_{\text {e }}$ and $\left\langle J_{\text {cnds }}\right\rangle=\left\langle J_{\text {evap }}^{\text {sp }}\right\rangle$, and hence the evaporation coefficient is equal to the condensation coefficient in the equilibrium state, i.e., $\alpha_{e}=\alpha_{c}$. In Sec. IV $\mathrm{C}$, we shall present the relation between the evaporation coefficient defined by Eq. (3) and the temperature in the bulk condensed phase for water and methanol [Eq. (13) is not used]. The result for water qualitatively agrees with that obtained in Ref. 10. However, in Ref. 10, Eq. (13) was evaluated in vapor-liquid equilibrium MD simulations by counting the number of molecules reflected from the interface and using the mass conservation law,

$$
\left\langle J_{\text {cnds }}\right\rangle=\left\langle J_{\text {coll }}\right\rangle-\left\langle J_{\text {ref }}\right\rangle,
$$

where $\left\langle J_{\text {ref }}\right\rangle=\left\langle J_{\text {out }}\right\rangle-\left\langle J_{\text {evap }}^{\text {sp }}\right\rangle$ is a reflected mass flux at the interface (see Fig. 1). The result therefore includes some ambiguity arising from the definition of the reflected molecule. As mentioned earlier, the complete classification of every individual molecule outgoing from the interface as either evaporated or reflected one in a vapor-liquid two-phase system may be impossible. This is because the reflected molecules in many cases once condense into the inside of the interface before being reemitted, and therefore it is difficult to define the reflected molecule without ambiguity. Our method is free from such ambiguity thanks to the analysis based on the virtual vacuum simulation.

\section{COMPUTATIONAL METHOD}

We execute MD simulations for vapor-liquid equilibrium states and nonequilibrium steady states of evaporation into a virtual vacuum. Several cases of the temperature of the

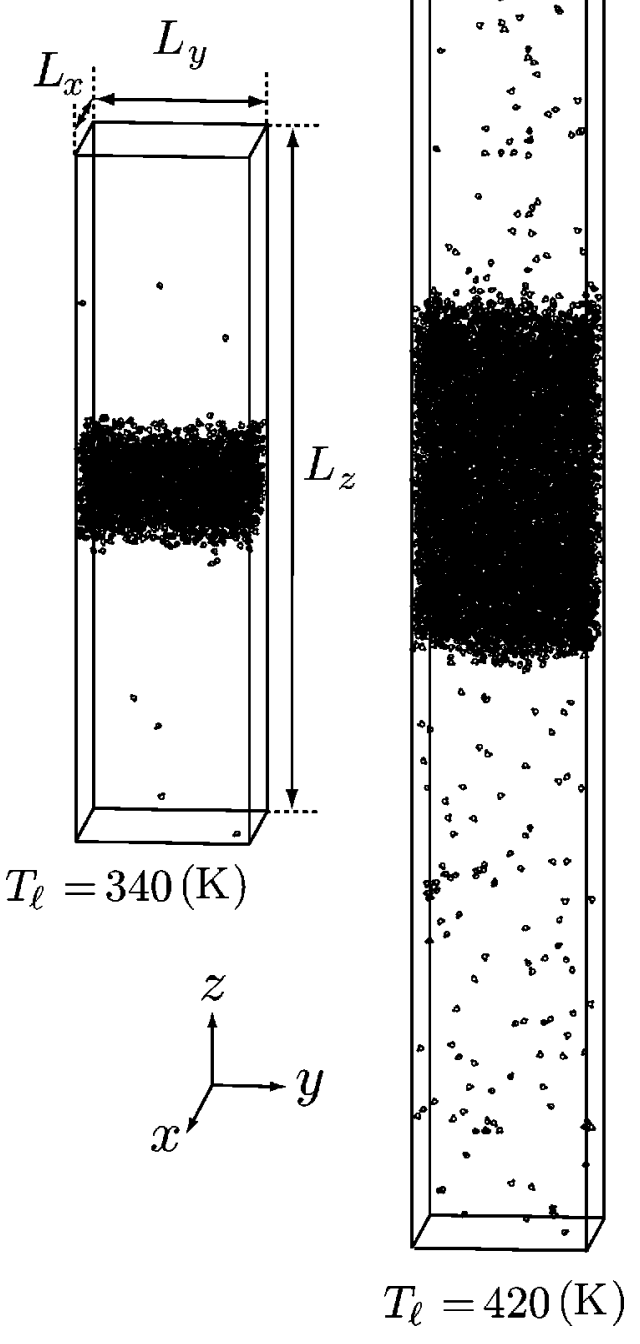

FIG. 2. Snapshots of equilibrium simulations of water.

liquid phase $T_{\ell}$ are computed: $300 \mathrm{~K} \leqslant T_{\ell} \leqslant 460 \mathrm{~K}$ for water and $260 \mathrm{~K} \leqslant T_{\ell} \leqslant 390 \mathrm{~K}$ for methanol. Typical examples for equilibrium states are shown in Fig. 2. The computational method is as a whole the same as that used in the previous study for monatomic molecule. ${ }^{6}$ In this section, we shall explain an intermolecular potential used in the present simulations for polyatomic molecules at some length, and then summarize the features of the methods for equilibrium and virtual vacuum simulations. To make the present paper selfcontained, we do not suppress the important information in the computational method, even though it is described in Ref. 6.

\section{A. Intermolecular potential}

MD simulation numerically solves the equations of motions for molecules in a simulation cell (see Fig. 2). We 
TABLE I. The parameters of TIP3P model for water.

\begin{tabular}{|c|c|c|c|c|c|}
\hline \multicolumn{2}{|c|}{ Geometry of molecule } & \multicolumn{2}{|c|}{ Lennard-Jones parameters } & \multicolumn{2}{|c|}{ Partial charges } \\
\hline$r_{\mathrm{OH}}(\AA)$ & $\angle \mathrm{HOH}$ & $\sigma_{\mathrm{OO}}(\AA)$ & $\epsilon_{\mathrm{OO}}(\mathrm{K})$ & $z_{\mathrm{O}}$ & $z_{\mathrm{H}}$ \\
\hline 0.9572 & $104.52^{\circ}$ & 3.1507 & 76.5289 & -0.834 & 0.417 \\
\hline
\end{tabular}

integrate the equations of motions transformed with the quaternion algorithm ${ }^{11}$ by the leap-frog method with the time step 0.5 fs. As the intermolecular potential, we adopt transferable intermolecular potential with three points (TIP3P) ${ }^{12}$ for water and optimized potential for liquid simulations $(\mathrm{OPLS})^{13}$ for methanol, since they are widely used ${ }^{14,15}$ and the computation time is not so large; we have to run many simulations to obtain a large number of samples for the accurate construction of the distribution function.

Both potentials are three-site rigid models consisted of Lennard-Jones 12-6 potential and Coulomb potential. According to these models, the intermolecular potential between a site $m$ of a molecule and a site $n$ of a different molecule can be written as

$$
\begin{aligned}
\phi_{m n}= & 4 \epsilon_{m n}\left[\left(\frac{\sigma_{m n}}{r_{m n}}\right)^{12}-\left(\frac{\sigma_{m n}}{r_{m n}}\right)^{6}\right] \\
& +\frac{z_{m} e_{0} z_{n} e_{0}}{4 \pi \epsilon_{0}}\left[\frac{1}{r_{m n}}+\frac{r_{m n}}{r_{\text {cut }}^{2}}-\frac{2}{r_{\text {cut }}}\right],
\end{aligned}
$$

where $r_{m n}$ is the distance between the site $m$ and the site $n$, $z_{m} e_{0}$ and $z_{n} e_{0}$ are partial charges for the sites, $e_{0}$ is the elementary charge, $\epsilon_{0}$ is the dielectric constant of vacuum, and $\epsilon_{m n}$ and $\sigma_{m n}$ are the usual Lennard-Jones parameters (see Tables I and II). As indicated in Eq. (15), the electrostatic term is shifted and scaled smoothly to zero at $r_{\text {cut }}(0.9 \mathrm{~nm})$, and lattice summation techniques are not used. The applicability of the shifted and scaled Coulomb potential has been confirmed in Ref. 16, and it is known that the use of the Ewald summation to the vapor-liquid two-phase system leads to some unnatural behaviors. ${ }^{17}$ The Lennard-Jones part also is truncated at the same cutoff distance $r_{\text {cut }}$.

The molecular geometries of TIP3P water and OPLS methanol are shown in Fig. 3, where the symbol $\sim$ denotes the body-fixed principal axes on which the inertial tensor is diagonal. Since TIP3P water and OPLS methanol are nonlinear three-site rigid models, the number of the internal degrees of freedom of a molecule is $n=3$, and $E$ in Eq. (5) is the internal energy associated with rotational motions, written as

$$
E=\frac{I_{\tilde{x}} \omega_{\tilde{x}}^{2}+I_{\tilde{y}} \omega_{\tilde{y}}^{2}+I_{\tilde{z}} \omega_{\tilde{z}}^{2}}{2},
$$

where $I_{j}$ and $\omega_{j}(j=\tilde{x}, \tilde{y}, \tilde{z})$ are, respectively, the principal moment of inertia and the angular velocity around the $j$ axis indicated in Fig. 3. The principal moments of inertia $I_{\widetilde{x}}, I_{\tilde{y}}$, and $I_{\tilde{z}}$ are, respectively, $1.77,1.16$, and $0.61\left(\mathrm{~g} \AA^{2} / \mathrm{mol}\right)$ for TIP3P water, and $17.47,16.73$, and $0.74\left(\mathrm{~g} \AA^{2} / \mathrm{mol}\right)$ for OPLS methanol.

\section{B. Equilibrium simulation}

In the equilibrium simulations, the periodic boundary conditions are imposed for all three directions of the simulation cell with dimensions $L_{x} \times L_{y} \times L_{z}$ (see Fig. 2). For both cases of water and methanol, in lower temperature cases of $T_{\ell} \leqslant 350 \mathrm{~K}$, we set $L_{x} \times L_{y} \times L_{z}=50 \times 50 \times 200 \AA^{3}$, in which $N=2000$ molecules are contained. At higher temperatures $T_{\ell} \geqslant 360 \mathrm{~K}$, we increase $L_{z}$ to $400 \AA$ and $N$ to 4000 .

After equilibrating the system, we continue the simulation for $5 \mathrm{~ns}$ and accumulate the configurations of all molecules in the cell every $1 \mathrm{ps}$. Since the system has two interfaces, the number of samples $N_{s}=10000$. The ensemble averages for various macroscopic quantities can be evaluated from $N_{s}$ sampled configurations. For example, an averaged density is calculated by

$$
\rho=\frac{1}{N_{s} \Xi_{p}} \sum_{N_{s}} \sum_{i \in \Xi_{p}} m^{i}
$$

where $\Xi_{p}$ is a volume element in the physical space, $m^{i}$ is the mass of the $i$ th molecule (all molecules have the same mass), and $\Sigma_{i \in \Xi_{p}} m^{i}$ denotes the summation of the mass of the molecules whose center of mass are contained in $\Xi_{p}$.

The averaged bulk vapor density (saturated vapor density) $\rho_{v}$ and bulk liquid density $\rho_{\ell}$ of TIP3P water and OPLS methanol obtained from the equilibrium simulations are shown in Tables III and IV. As can be seen from the tables,

\begin{tabular}{|c|c|c|c|c|c|c|c|c|c|}
\hline \multicolumn{3}{|c|}{ Geometry of molecule } & \multicolumn{4}{|c|}{ Lennard-Jones parameters } & \multicolumn{3}{|c|}{ Partial charges } \\
\hline$r_{\mathrm{MO}}(\AA)$ & $r_{\mathrm{OH}}(\AA)$ & $\angle \mathrm{MOH}$ & $\sigma_{\mathrm{MM}}(\AA)$ & $\epsilon_{\mathrm{MM}}(\mathrm{K})$ & $\sigma_{\mathrm{OO}}(\AA)$ & $\epsilon_{\mathrm{OO}}(\mathrm{K})$ & $z_{\mathrm{O}}$ & $z_{\mathrm{H}}$ & $z_{\mathrm{M}}$ \\
\hline 1.4246 & 0.9451 & $108.53^{\circ}$ & 3.775 & 104.155 & 3.071 & 85.513 & -0.700 & 0.435 & 0.265 \\
\hline
\end{tabular}
TIP3P and OPLS models give large values of saturated vapor density compared with experimental ones. In a preliminary

TABLE II. The parameters of OPLS model for methanol. 

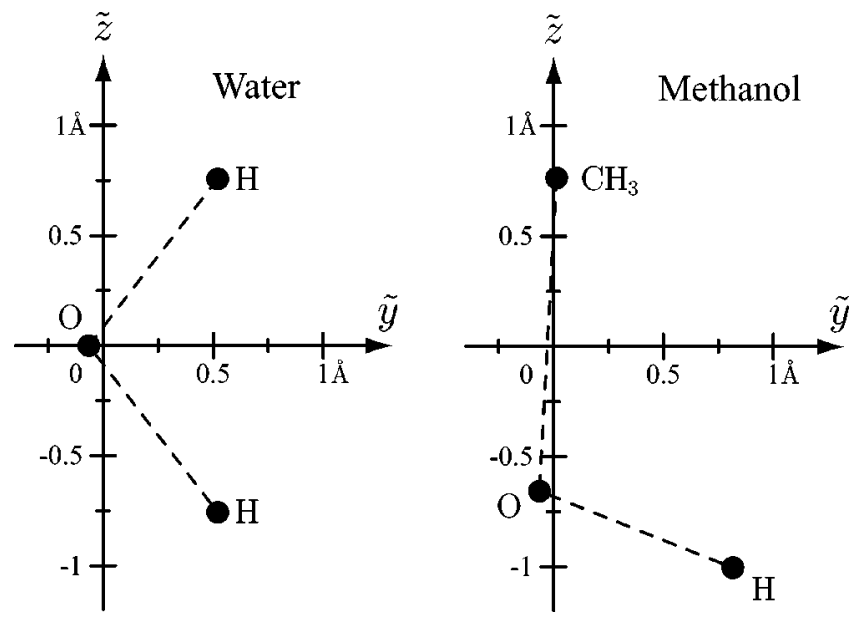

FIG. 3. Molecular geometries of TIP3P water and OPLS methanol molecules. The origin is the center of mass of a molecule, and $\tilde{x}, \tilde{y}$, and $\tilde{z}$ axes correspond to the principal axes of inertia, where $\tilde{x}$ axes is perpendicular to the $\tilde{y z}$ plane.

computation, we have confirmed that the use of a six-site model for methanol ${ }^{18}$ does not lead to a sufficient improvement for saturated vapor density and requires more CPU time than OPLS, and hence we have decided to use OPLS.

According to Ref. 19, we can estimate the critical density and critical temperature for both models from the values of $\rho_{v}$ and $\rho_{\ell}$ in Tables III and IV. The critical density $\rho_{\text {cr }}$ $=0.31 \mathrm{~g} / \mathrm{cm}^{3}$ for TIP3P water and $0.27 \mathrm{~g} / \mathrm{cm}^{3}$ for OPLS methanol agree with experimental values $0.32 \mathrm{~g} / \mathrm{cm}^{3}$ for water and $0.27 \mathrm{~g} / \mathrm{cm}^{3}$ for methanol, respectively. ${ }^{20,21}$ The estimated critical temperatures are $T_{\mathrm{cr}}=515.9 \mathrm{~K}$ for TIP3P water and $403.7 \mathrm{~K}$ for OPLS methanol, which are small compared with the corresponding experimental values $647.1 \mathrm{~K}$ and $512.6 \mathrm{~K}^{20,21}$ Therefore, the present results are not directly compared with experimental ones. In Sec. IV, to compensate the disagreements of saturated vapor density and the critical temperature, the results are arranged in terms of a normalized temperature $T / T_{\mathrm{cr}}$.

The spatial density distributions in the vicinity of the interface are shown in Fig. 4. The density profiles in equilibrium state are well fitted to a hyperbolic tangent function

$$
\rho(z)=\frac{\rho_{v}+\rho_{\ell}}{2}+\frac{\rho_{v}-\rho_{\ell}}{2} \tanh \left(\frac{z-Z_{m}}{0.455 \delta}\right)
$$

where $Z_{m}$ and $\delta$ are, respectively, the center of the transition layer and the 10-90 thickness (see Tables III and IV), which are estimated by a least-squares method.

\section{Virtual vacuum simulation}

In the virtual vacuum simulations, we introduce a perfectly absorbing boundary at a distance from the interface and eliminate molecules there (Fig. 5), while the periodic boundary conditions are applied in the $x$ and $y$ directions. Molecules evaporate into the virtual vacuum through the perfectly absorbing boundary and the interface recedes with time as a result of the evaporation. The steady evaporation state is realized on the moving coordinate

$$
z^{*}=\frac{z-\left(Z_{m}-v_{s} t\right)}{\delta}, \quad v_{s}=\frac{J_{s}}{\rho_{\ell}}
$$

where $t$ is the time from the beginning of the virtual vacuum simulation, $J_{s}$ is a molecular flux evaporating into virtual vacuum, and $v_{s}$ is the speed of the moving coordinate. The averaged values for macroscopic quantities and the distribution functions are evaluated on the moving coordinate $z^{*}$. The evaporation flux $J_{s}$ is estimated at each time step at $z^{*}$ $=L_{g}^{*}$, where we set the perfectly absorbing boundary (see Fig. $5)$.

The control of the temperature in the bulk liquid phase is important for the realization of the steady evaporation state. Using the velocity scaling method, ${ }^{11}$ we control the temperature of the liquid phase in the region $z^{*}<-L_{c}^{*}$ as shown schematically in Fig. 5. As the result, the averaged temperature of the bulk liquid phase is kept almost uniform and constant at a specified $T_{\ell}$. Anisimov et al. have executed a similar virtual vacuum simulation for monatomic molecules. ${ }^{22}$ However, since they have controlled not the temperature in the bulk liquid phase but the temperature at the floor of MD cell, a large temperature gradient has appeared in the bulk liquid phase (see Fig. 3 in Ref. 22). This leads to an unfavorable situation where one cannot determine the reference temperature uniquely.

TABLE III. The results of equilibrium and virtual vacuum simulations for water. The values in parentheses in $\rho_{v}$ and $\rho_{\ell}$ columns are experimental ones, and

\begin{tabular}{|c|c|c|c|c|c|c|c|c|c|}
\hline$T_{\ell}\left(T_{\ell} / T_{\mathrm{cr}}\right)(\mathrm{K})$ & $\rho_{v}\left(10^{-3} \mathrm{~g} / \mathrm{cm}^{3}\right)$ & $\rho_{\ell}\left(\mathrm{g} / \mathrm{cm}^{3}\right)$ & $\delta(\AA)$ & $\langle J\rangle_{\mathrm{e}}\left[\mathrm{g} /\left(\mathrm{cm}^{2} \mathrm{~s}\right)\right]$ & $\left\langle J_{\text {evap }}^{\mathrm{sp}}\right\rangle\left[\mathrm{g} /\left(\mathrm{cm}^{3} \mathrm{~s}\right)\right]$ & $\left\langle J_{\mathrm{evap}}^{\mathrm{sp}}\right\rangle_{\mathrm{c}}\left[\mathrm{g} /\left(\mathrm{cm}^{3} \mathrm{~s}\right)\right]$ & $\alpha_{e}$ & $\rho_{c}\left(10^{-3} \mathrm{~g} / \mathrm{cm}^{3}\right)$ & $\alpha \rho_{v} / 2\left(10^{-3} \mathrm{~g} / \mathrm{cm}^{3}\right)$ \\
\hline $300(0.581)$ & $0.07(0.03)$ & $0.95(1.00)$ & 5.31 & 1.03 & 1.02 & 0.00 & 0.99 & 0.032 & 0.034 \\
\hline 340 (0.659) & $0.51(0.17)$ & $0.91(0.98)$ & 6.13 & 8.10 & 7.41 & 0.41 & 0.92 & 0.24 & 0.23 \\
\hline $360(0.698)$ & $1.01(0.38)$ & $0.89(0.97)$ & 6.73 & 16.51 & 14.41 & 1.11 & 0.87 & 0.47 & 0.44 \\
\hline $380(0.737)$ & $2.18(0.75)$ & $0.86(0.95)$ & 8.04 & 36.38 & 29.47 & 3.83 & 0.81 & 1.01 & 0.88 \\
\hline $400(0.775)$ & $3.73(1.37)$ & $0.83(0.94)$ & 8.97 & 63.89 & 49.89 & 7.18 & 0.78 & 1.72 & 1.46 \\
\hline $420(0.814)$ & $6.54(2.35)$ & $0.79(0.92)$ & 10.03 & 114.89 & 81.47 & 15.97 & 0.71 & 2.87 & 2.32 \\
\hline $440(0.853)$ & $11.50(3.83)$ & $0.76(0.90)$ & 11.44 & 206.85 & 128.40 & 33.41 & 0.62 & 4.42 & 3.57 \\
\hline $460(0.892)$ & 20.08 (5.98) & $0.71(0.88)$ & 13.15 & 369.21 & 184.10 & 57.70 & 0.50 & 6.10 & 5.01 \\
\hline
\end{tabular}
$\langle J\rangle_{\mathrm{e}}=\rho_{v} \sqrt{R T_{\ell} /(2 \pi)}$ is the outgoing (or incoming) molecular mass flux in the equilibrium state at $T_{\ell}$. 
TABLE IV. The results of equilibrium and virtual vacuum simulations for methanol. The values in parentheses in $\rho_{v}$ and $\rho_{\ell}$ columns are experimental ones, and $\langle J\rangle_{\mathrm{e}}=\rho_{v} \sqrt{R T_{\ell} /(2 \pi)}$ is the outgoing (or incoming) molecular mass flux in the equilibrium state at $T_{\ell}$.

\begin{tabular}{|c|c|c|c|c|c|c|c|c|c|}
\hline$T_{\ell}\left(T_{\ell} / T_{\mathrm{cr}}\right)(\mathrm{K})$ & $\rho_{v}\left(10^{-3} \mathrm{~g} / \mathrm{cm}^{3}\right)$ & $\rho_{\ell}\left(\mathrm{g} / \mathrm{cm}^{3}\right)$ & $\delta(\AA)$ & $\langle J\rangle_{\mathrm{e}}\left[\mathrm{g} /\left(\mathrm{cm}^{2} \mathrm{~s}\right)\right]$ & $\left\langle J_{\mathrm{evap}}^{\mathrm{sp}}\right\rangle\left[\mathrm{g} /\left(\mathrm{cm}^{3} \mathrm{~s}\right)\right]$ & $\left\langle J_{\mathrm{evap}}^{\mathrm{sp}}\right\rangle_{\mathrm{c}}\left[\mathrm{g} /\left(\mathrm{cm}^{2} \mathrm{~s}\right)\right]$ & $\alpha_{e}$ & $\rho_{c}\left(10^{-3} \mathrm{~g} / \mathrm{cm}^{3}\right)$ & $\alpha \rho_{v} / 2\left(10^{-3} \mathrm{~g} / \mathrm{cm}^{3}\right)$ \\
\hline $260(0.644)$ & $0.14(0.025)$ & $0.75(0.82)$ & 7.01 & 1.41 & 1.25 & 0.08 & 0.88 & 0.060 & 0.060 \\
\hline $300(0.743)$ & $1.40(0.25)$ & $0.70(0.78)$ & 8.73 & 15.60 & 11.21 & 1.34 & 0.72 & 0.61 & 0.50 \\
\hline $310(0.768)$ & $2.21(0.39)$ & $0.69(0.78)$ & 9.25 & 25.06 & 17.19 & 3.48 & 0.69 & 0.95 & 0.76 \\
\hline $320(0.793)$ & $2.99(0.61)$ & $0.67(0.77)$ & 9.87 & 34.41 & 22.56 & 5.54 & 0.66 & 1.32 & 0.98 \\
\hline $330(0.817)$ & $4.68(0.91)$ & $0.66(0.76)$ & 10.50 & 54.67 & 34.14 & 8.42 & 0.62 & 1.82 & 1.46 \\
\hline $340(0.842)$ & $7.32(1.33)$ & $0.64(0.75)$ & 11.35 & 86.77 & 49.01 & 12.84 & 0.56 & 2.63 & 2.07 \\
\hline $350(0.867)$ & $10.16(1.91)$ & $0.62(0.74)$ & 12.29 & 122.17 & 66.61 & 22.18 & 0.55 & 3.73 & 2.77 \\
\hline $360(0.892)$ & $13.85(2.67)$ & $0.60(0.72)$ & 13.58 & 168.95 & 82.55 & 31.70 & 0.49 & 4.81 & 3.38 \\
\hline $370(0.917)$ & $19.56(3.68)$ & $0.58(0.71)$ & 15.41 & 241.80 & 98.70 & 38.10 & 0.41 & 6.24 & 3.99 \\
\hline $380(0.941)$ & $29.86(4.98)$ & $0.54(0.70)$ & 17.55 & 374.12 & 128.89 & 51.29 & 0.34 & 7.85 & 5.14 \\
\hline $390(0.966)$ & $45.74(6.65)$ & $0.51(0.69)$ & 20.42 & 580.56 & 160.04 & 63.14 & 0.28 & 9.94 & 6.30 \\
\hline
\end{tabular}

In the previous paper, ${ }^{6}$ we have verified that the results of virtual vacuum simulations are hardly affected by the choices of $L_{g}^{*}$ and $L_{c}^{*}$, if $L_{g}^{*}$ and $L_{c}^{*}$ are in the ranges of 2 $\leqslant L_{g}^{*} \leqslant 4$ and $0 \leqslant L_{c}^{*} \leqslant 1$, respectively. In the present study, we set $L_{g}^{*}=4$ and $L_{c}^{*}=1$ as in the previous paper. ${ }^{23}$

The virtual vacuum simulation is started from a configuration obtained in the equilibrium simulation. At the time when the number of molecules in a region $z^{*}<L_{g}$ decreases to 1000 due to the evaporation into virtual vacuum, we stop the simulation to avoid sampling erroneous configurations. Since this leads to the shortage of the number of samples for ensemble averages, we run seven more simulations starting from different initial conditions. ${ }^{6}$

\section{NUMERICAL RESULTS AND DISCUSSION}

In this section, we shall present the numerical results for the virtual vacuum simulation. We discuss the detailed property of evaporation into virtual vacuum, thereby clarifying the characteristics of the distribution function of evaporating molecules in the case of water and methanol.
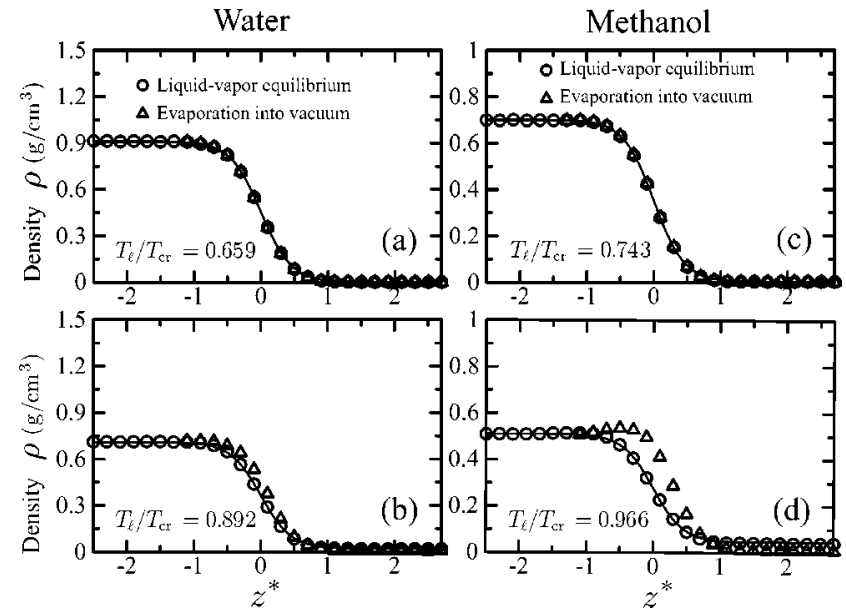

FIG. 4. The profiles of the averaged density defined by Eq. (17) in the vicinity of the interface $\left(\Xi_{p}=0.2 L_{x} L_{y} \delta\right)$. The abscissa $z^{*}$ is a normalized coordinate defined by Eq. (19). The solid line represents the fitting function given by Eq. (18).

\section{A. Spontaneous-evaporation flux}

We shall start with the verification of steadiness of the evaporation into virtual vacuum for water and methanol, because the realization of the steady evaporation state implies the existence of the spontaneous-evaporation mass flux $\left\langle J_{\text {evap }}^{\mathrm{sp}}\right\rangle$ determined by the bulk liquid temperature $T_{\ell}$ only. The time evolution of a mass flux passing through the perfectly absorbing boundary $z^{*}=L_{g}^{*}$ is shown in Fig. 6 . In the figure, $\left\langle J_{+}\right\rangle$is a mass flux in the positive $z^{*}$ direction averaged for a small time interval. In the cases of lower temperature [Figs. 6(a) and 6(c)], an almost steady molecular mass flux is generated from the beginning of the virtual vacuum simulation, as in the case of $85 \mathrm{~K}$ for argon (see Fig. 5 in Ref. 6). Even for a higher temperature case [Fig. 6(d)], one can see that an almost steady state is established after an initial transient state dies out, which was also observed at $130 \mathrm{~K}$ in the case of argon. It is worth noting that $\left\langle J_{\text {out }}\right\rangle_{\mathrm{e}}$ is almost equal to or larger than $\left\langle J_{+}\right\rangle_{z^{*}=L_{g}^{*}}$ at lower $T_{\ell}$, and the difference between them increases more and more as $T_{\ell}$ increases.

After the steady state is reached, an averaged net mass flux $\left\langle J_{+}\right\rangle-\left\langle J_{-}\right\rangle$is calculated at various points on the moving coordinate, where $\left\langle J_{+}\right\rangle-\left\langle J_{-}\right\rangle$denotes the difference of mass fluxes in the positive and negative $z^{*}$ directions, which are averaged for a sufficiently long time interval in the steady state. The spatial uniformity of the net mass flux is clearly shown in Fig. 7. We can therefore define the spontaneousevaporation flux by the net flux in the virtual vacuum simu-

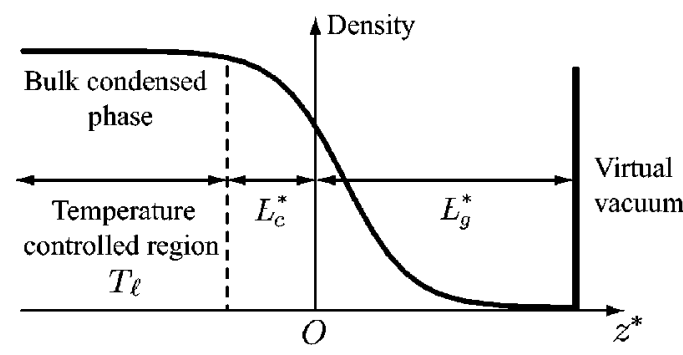

FIG. 5. Schematic of simulation of evaporation into the virtual vacuum on the moving coordinate $z^{*}$ defined by Eq. (19). 

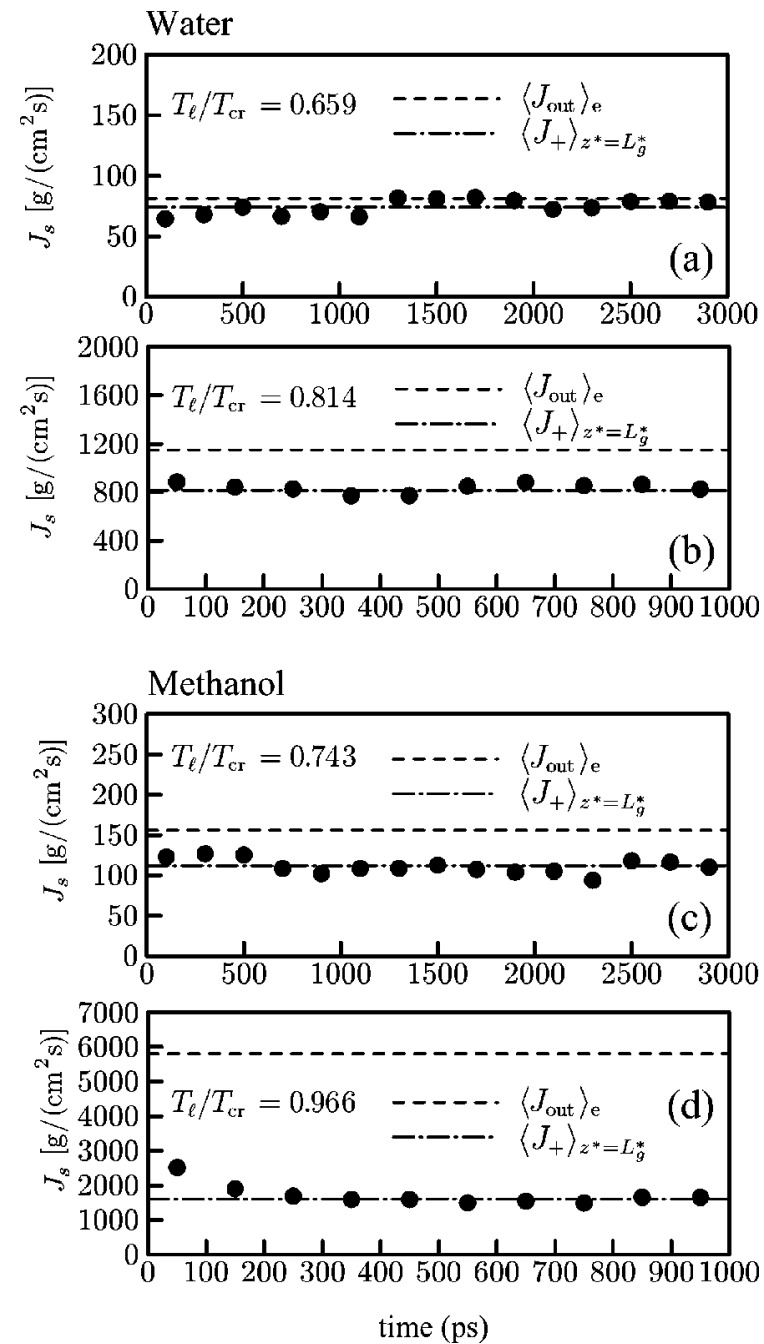

FIG. 6. The temporal evolution of evaporating mass flux in virtual vacuum simulations $\left(L_{g}^{*}=4\right)$ are shown by the black circles, evaluated by the time average for a small time interval between adjacent two symbols. The dashed line denotes $\left\langle J_{\text {out }}\right\rangle_{e}$, the outgoing mass flux of molecules in the equilibrium state, and the dash-dotted line denotes the outgoing mass flux in the steady state of evaporation into virtual vacuum.

lation, i.e., $\left\langle J_{\text {evap }}^{\text {sp }}\right\rangle=\left\langle J_{+}\right\rangle-\left\langle J_{-}\right\rangle$. The density of vapor evaporating into virtual vacuum $\rho_{c}$ and the spontaneous-evaporation flux $\left\langle J_{\text {evap }}^{\text {sp }}\right\rangle$ in the steady state are tabulated in Tables III and IV.

\section{B. Cluster formation}

In Fig. 8, the trajectories of molecules evaporating into virtual vacuum in the steady state are shown, where a solid line represents the projection of the trajectory of center of mass of a molecule into the $z t$ plane and the time $t=0$ is taken at an arbitrary time in the steady state. In the cases of lower temperature [Figs. 8(a) and 8(c)], all molecules evaporated from the condensed phase go straight toward the virtual vacuum, while in the higher temperature cases [Figs. 8(b) and $8(\mathrm{~d})$ ], there are several molecules that move toward the condensed phase. This is caused by the molecular interaction in the vapor phase. Furthermore, some molecules in double or triple helical motions can be seen in the figure. Such
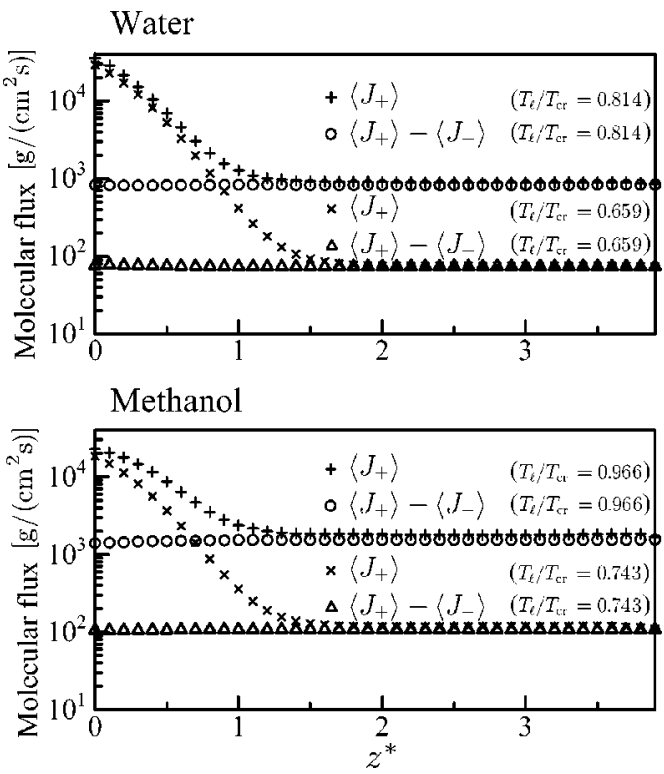

FIG. 7. The spatial distribution of outgoing mass flux $\left\langle J_{+}\right\rangle$and net mass flux $\left\langle J_{+}\right\rangle-\left\langle J_{-}\right\rangle$in the steady state of evaporation into virtual vacuum.

groups are referred to as clusters. Notice that the clusters are not formed in the vapor region but emitted from the interface.

Hill $^{24}$ defined a cluster as a collection of monomers that are each energetically bound to at least one other monomer. According to his definition, the $i$ th molecule is regarded as a part of a cluster when the sum of the relative kinetic energy and the potential energy to any $j$ th molecule $(i \neq j)$ is negative,

$$
\frac{\left(\boldsymbol{p}_{i}-\boldsymbol{p}_{j}\right)^{2}}{2 m^{i}}+U_{i j}<0, \quad U_{i j}=\sum_{m}^{\text {on } i \text { on } j} \sum_{n} \phi_{m n},
$$

where $\boldsymbol{p}_{i}$ is the momentum of the $i$ th molecule, and $U_{i j}$ is the potential energy between the $i$ th and $j$ th molecules. Using this definition, we can define an evaporating cluster mass flux by counting the number of molecules evaporating into virtual vacuum with satisfying the condition Eq. (20). Figure 9 shows the ratio of the average of the evaporating cluster mass flux $\left\langle J_{\text {evap }}^{\text {sp }}\right\rangle_{\mathrm{c}}$ to the average of the spontaneousevaporation mass flux $\left\langle J_{\text {evap }}^{\text {sp }}\right\rangle$, where the result for argon is also plotted. One can see that the ratio $\left\langle J_{\text {evap }}^{\text {sp }}\right\rangle_{\mathrm{c}} /\left\langle J_{\text {evap }}^{\text {sp }}\right\rangle$ increases as the bulk liquid temperature $T_{\ell}$ approaches the
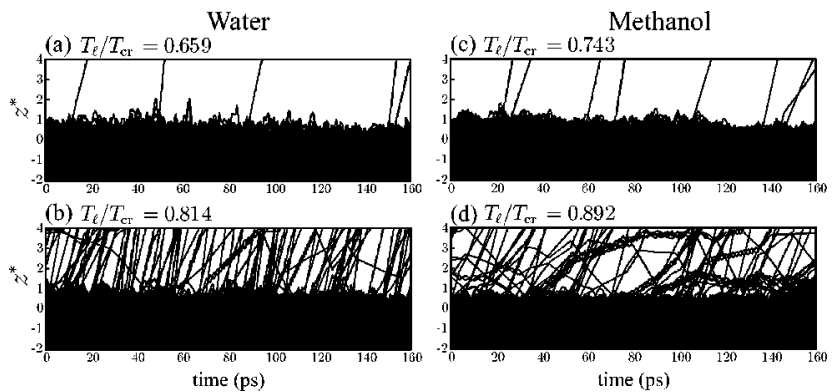

FIG. 8. Trajectories of molecules evaporating into virtual vacuum in the vicinity of the interface. 


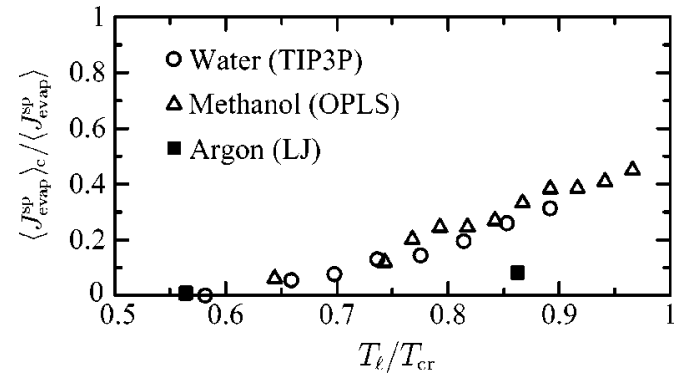

FIG. 9. Ratio of the cluster mass flux evaporated into virtual vacuum to spontaneous-evaporation mass flux.

critical temperature. This may be due to the strong hydrogen bonding. ${ }^{15}$ In fact, as shown in Fig. 9, the argon clusters are rarely formed even in high temperature cases. The cluster formation is a characteristic feature of associating fluids such as water and methanol.

\section{Evaporation coefficient}

Since we have already obtained $\left\langle J_{\text {evap }}^{\text {sp }}\right\rangle$ as a function of $T_{\ell}$ in Sec. IV A, the evaporation coefficient $\alpha_{e}$ can easily be evaluated from Eq. (3). The results for water and methanol are presented in Fig. 10, together with those by other authors and those for argon by the present authors ${ }^{6}$ (see also Tables III and IV). Note that, in the previous paper ${ }^{6}$ and in other papers, ${ }^{10,25} \alpha_{e}$ is called the condensation coefficient in the equilibrium state (the reason is explained in Sec. II).

Although theoretical and experimental studies have so far reported various values of the evaporation coefficient of water from $10^{-4}$ to unity at near room temperature $\left(T_{\ell} / T_{\mathrm{cr}}\right.$ $\approx 0.5$ ) (see Ref. 26 ), our result shows that $\alpha_{e}$ of water is close to unity at around $T_{\ell} / T_{\mathrm{cr}} \approx 0.6$ and slowly decreases with increase in $T_{\ell}$. The result for methanol is almost the same as that for water. In the latest experiment for condensation of methanol vapor in a shock tube by Fujikawa et al., ${ }^{27}$ they reported that $\alpha_{e}$ of methanol near equilibrium state was nearly unity at $T_{\ell} / T_{\mathrm{cr}}=0.57$.

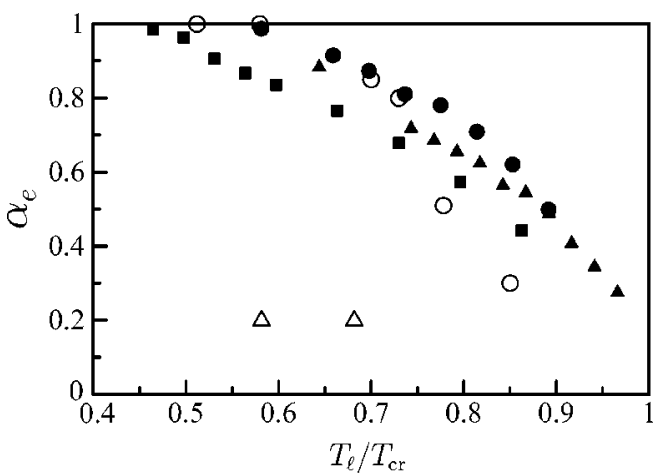

$$
\begin{aligned}
& \text { - Water (present MD) } \\
& \text { Methanol (present MD) } \\
& \text { Argon (Ishiyama et al., 2003) } \\
& \text { O Water (Nagayama and Tsuruta, 2002) } \\
& \triangle \text { Methanol (Matsumoto et al., 1998) }
\end{aligned}
$$

FIG. 10. Evaporation coefficient $\alpha_{e}$. Open circles are the results in Ref. 10, and open triangles those in Ref. 25.
Let us compare our results with those of other molecular dynamics studies. Nagayama and Tsuruta ${ }^{10}$ calculated the condensation coefficient $\alpha_{c}$ of water. Their results agree with ours (see Fig. 10), although their definitions of $\alpha_{c}$ includes some ambiguity as mentioned in Sec. II. Matsumoto ${ }^{25}$ examined the condensation coefficient $\alpha_{c}$ for methanol, and reported that the values were about 0.2 (see Fig. 10) and the temperature dependence of $\alpha_{c}$ for methanol was not significant. In Ref. 25, they calculated the correlation of incident and outgoing molecules, and defined the reflected molecules as outgoing molecules that correlate with incident molecules strongly. Therefore, their definition of reflected molecules (and hence the definition of $\alpha_{c}$ ) also contains some uncertainty.

As mentioned in Sec. II, an evaporated molecule and a reflected one may be indistinguishable in a vapor-liquid coexistence state. The only clearly defined quantities are the mass flux and distribution function of molecules evaporating into virtual vacuum, except for outgoing and incoming molecules. The concept of reflection, such as an incident molecule bounces back on a surface, which has been implicitly assumed in the previous studies, ${ }^{10,25}$ inevitably contains uncertainties.

\section{Distribution functions for translational and internal motions}

Now, we shall evaluate the distribution function of the translational velocity of molecules evaporating into virtual vacuum, $\hat{f}_{\text {trans. In }}$ MD simulations, the distribution function of the translational velocity of the center of mass of molecule can be calculated by

$$
\hat{f}_{\text {trans }}=\hat{f}_{x} \hat{f}_{y} \hat{f}_{z}, \hat{f}_{j}=\frac{1}{\rho N_{s} \Xi_{p} \Xi_{v}^{j}} \sum_{N_{s}} \sum_{i \in\left(\Xi_{p} \cap \Xi_{v}^{j}\right)} m^{i},
$$

where $\hat{f}_{j}$ is the marginal distribution of the $j$ component of translational velocity $(j=x, y, z), \Xi_{v}^{j}$ is a one-dimensional volume element in the $j$ direction in the three-dimensional molecular velocity space, and $\Xi_{p} \cap \Xi_{v}^{j}$ denotes a fourdimensional volume element in the six-dimensional phase space. Here, we recall that $\hat{f}_{\text {trans }}$ signifies that the distribution function is normalized by the density. Equation (21) assumes that $\xi_{x}, \xi_{y}$, and $\xi_{z}$ are the independent random variables. As a check of a necessary condition for statistical independence, it is confirmed that the correlation coefficients between them are small compared with unity.

In Fig. 11, the velocity distributions of molecules evaporating into virtual vacuum at $z^{*}=L_{g}^{*}$ are plotted for some temperatures. The abscissa $\zeta_{j}=\xi_{j} / \sqrt{2 R T_{\ell}}$ is the $j$ component of the normalized molecular velocity. Figure 11 shows that, for both water and methanol, the translational velocity distributions of $\zeta_{x}$ and $\zeta_{y}$ denoted by triangles and squares almost agree with a one-dimensional normalized Maxwellian $(1 / \sqrt{\pi}) \exp \left(-\zeta_{j}^{2}\right)$ denoted by a solid curve. However, the distributions for relatively high $T_{\ell}$ cases slightly shift to lower temperature distributions. The decrease of temperature of evaporating molecules will be discussed briefly in Sec. IV E. 

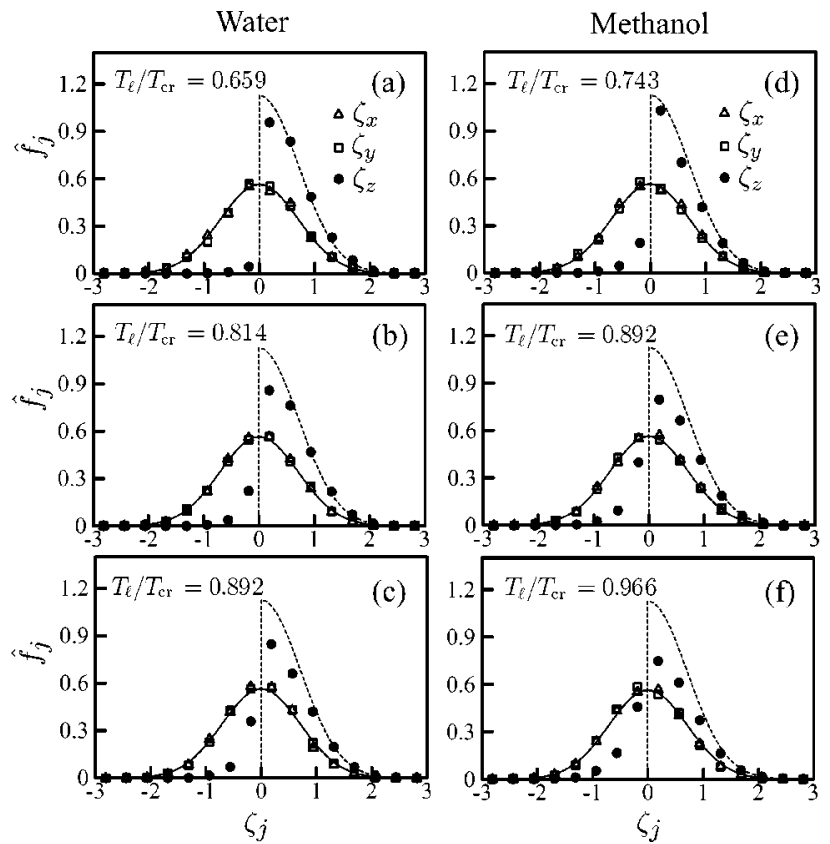

FIG. 11. Distribution functions of translational velocity of water and methanol molecules evaporating into virtual vacuum at $z^{*}=L_{g}^{*}=4$. The solid curve indicates a one-dimensional normalized Maxwellian $(1 / \sqrt{\pi}) \exp \left(-\zeta_{j}^{2}\right)$, and the dashed curve a one-dimensional normalized half-Maxwellian $(2 / \sqrt{\pi}) \exp \left(-\zeta_{j}^{2}\right)$ for $\zeta_{j}>0\left(\Xi_{p}=0.2 L_{x} L_{y} \delta\right.$ and $\left.\Xi_{v}^{j}=0.375 \sqrt{2 R T_{\ell}}\right)$.

For relatively low $T_{\ell}$ cases shown in Figs. 11(a) and 11(d), the velocity distribution of $\zeta_{z}$ denoted by closed circles becomes nearly_a one-dimensional normalized halfMaxwellian $(2 / \sqrt{\pi}) \exp \left(-\zeta_{j}^{2}\right)\left(\zeta_{z}>0\right)$ denoted by a dashed curve. Consequently, the distribution function of the translational velocity in the three-dimensional form may be written as

$$
\hat{f}_{\text {trans }}=2 \hat{f}^{*} \quad\left(\xi_{z}>0\right),
$$

at relatively low $T_{\ell}$, where $\hat{f}^{*}$ is defined by Eq. (4).

At relatively high $T_{\ell}$, on the other hand, the distribution function of $\zeta_{z}$ deviates from the half-Maxwellian. From the molecular trajectories shown in Figs. 8(b) and 8(d) in higher $T_{\ell}$ cases, one can see that some trajectories in the vapor region have negative gradient, which means that $\zeta_{z}<0$, due to the intermolecular collisions or the cluster formation. These complicated interactions results in the excitation of molecules with negative normal velocity. Furthermore, as shown in Fig. 9, the ratio $\left\langle J_{\text {evap }}^{\mathrm{sp}}\right\rangle_{\mathrm{c}} /\left\langle J_{\text {evap }}^{\mathrm{sp}}\right\rangle$ increases with increase in $T_{\ell}$, and this leads to the increase in the number of molecules with negative normal velocity. At the same time, the molecular interaction reduces the number of molecules whose normal velocities are positive and small [Figs. 11(c) and 11(f)].

The mean free path of saturated vapor is shown in Table $\mathrm{V}$, which is evaluated by the formula for equilibrium state of hard-sphere gas, $\ell=1 /\left[\sqrt{2} \pi \sigma^{2}\left(\rho_{v} / m\right)\right]$. The mean free path of the vapor evaporating into virtual vacuum may roughly be estimated by replacing $\rho_{v}$ in the formula with $\rho_{c}$, the density of vapor evaporating into the virtual vacuum, shown in Tables III and IV. From the comparison of the size of $L_{g}^{*}(=4$
TABLE V. The mean free math in saturated vapor for water and methanol, evaluated by the formula for equilibrium state of hard-sphere gas, $\ell$ $=1 /\left[\sqrt{2} \pi \sigma^{2}\left(\rho_{v} / m\right)\right]$, where $\sigma=2.641 \AA$ for water and $3.626 \AA$ for methanol (Ref. 28).

\begin{tabular}{crrrr}
\hline \hline & Water & & & \multicolumn{2}{c}{ Methanol } \\
\cline { 1 - 2 } \cline { 4 - 5 }$T_{\ell}(\mathrm{K})$ & $\ell(\AA)$ & & $T_{\ell}(\mathrm{K})$ & $\ell(\AA)$ \\
\hline 300 & 13864 & & 260 & 6687 \\
340 & 1884 & & 300 & 650 \\
360 & 951 & & 310 & 411 \\
380 & 443 & & 320 & 304 \\
400 & 259 & & 330 & 195 \\
420 & 148 & & 340 & 124 \\
440 & 84 & & 350 & 90 \\
460 & 48 & & 360 & 66 \\
& & 370 & 47 \\
& & 380 & 31 \\
& & 390 & 20 \\
\hline \hline
\end{tabular}

normalized by $\delta$ ) with the mean free path of the vapor in Table V, one can see that the molecular interaction cannot be disregarded near the interface in the high temperature cases, where $\delta$ is the thickness of the transition layer shown in Tables III and IV.

The deviation from the half-Maxwellian in high temperature case may be caused by the above-mentioned molecular interaction in the vapor phase and a local equilibration in the transition layer, as discussed in the last paragraph in Sec. IV in the previous paper. ${ }^{6}$

Next, we shall move on to the distribution function associated with the internal (rotational) motion of molecules evaporating into virtual vacuum, $\hat{g}_{a}$. The distribution function of the angular velocity around the principal axes of molecule can be calculated by

$$
\hat{g}_{a}=\hat{g}_{\tilde{x}} \hat{g}_{\hat{y}} \hat{g}_{\tilde{z}}, \hat{g}_{j}=\frac{1}{\rho N_{s} \Xi_{p} \Xi_{a}^{j}} \sum_{N_{s}} \sum_{i \in\left(\Xi_{p} \cap \Xi_{a}^{j}\right)} m^{i},
$$

where $\Xi_{a}^{j}$ is a one-dimensional volume element around the $j$ axis in the three-dimensional angular velocity space $(j$ $=\widetilde{x}, \tilde{y}, \widetilde{z})$. In Fig. 12, we plot the angular velocity distributions of molecules evaporating into virtual vacuum at $z^{*}=L_{g}^{*}$. The abscissa $\nu_{j}=\omega_{j} \sqrt{I_{j}} /\left(2 k T_{\ell}\right.$ in the figure denotes the normalized angular velocity component around the $j$ axis. As can be seen from Fig. 12, the angular velocity distributions are isotropic and are nearly the Maxwellians for all temperatures, although the vapor is in an extreme nonequilibrium condition, i.e., evaporation into virtual vacuum. It is also confirmed that the correlation coefficients between each component of angular velocity and translational velocity are small compared with unity.

Furthermore, we shall calculate a rotational energy distribution of molecules evaporating into virtual vacuum $\hat{g}_{\text {rot }}$ by 

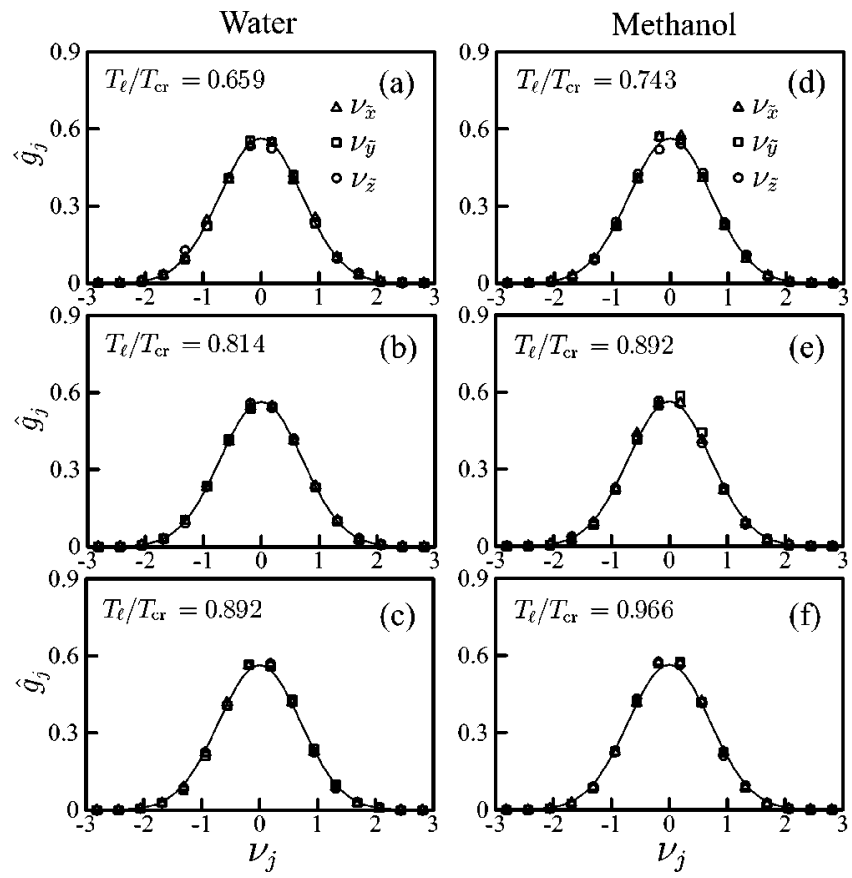

FIG. 12. Distribution functions of angular velocity around principal axes of water and methanol molecules evaporating into virtual vacuum at $z^{*}=L_{g}^{*}$ $=4$. The solid curve indicates a one-dimensional normalized Maxwellian $(1 / \sqrt{\pi}) \exp \left(-\nu_{j}^{2}\right)\left[\Xi_{p}=0.2 L_{x} L_{y} \delta\right.$ and $\left.\Xi_{a}^{j}=0.375 \sqrt{I_{j} /\left(2 k T_{\ell}\right)}\right]$.

$$
\hat{g}_{\mathrm{rot}}=\frac{1}{\rho N_{s} \Xi_{p} \Xi_{e}} \sum_{N_{s}} \sum_{i \in\left(\Xi_{p} \cap \Xi_{e}\right)} m^{i}
$$

where $\Xi_{e}$ is a one-dimensional volume element in the onedimensional rotational energy space. In Fig. 13, the rotational energy distributions of molecules evaporating into virtual vacuum at $z^{*}=L_{g}^{*}$ are presented, where the abscissa $\Theta$ $=E /\left(k T_{\ell}\right)$ denotes the normalized rotational energy. Figure 13 shows that the rotational energy distributions in the case of relatively high $T_{\ell}$ slightly shift to lower temperature dis-
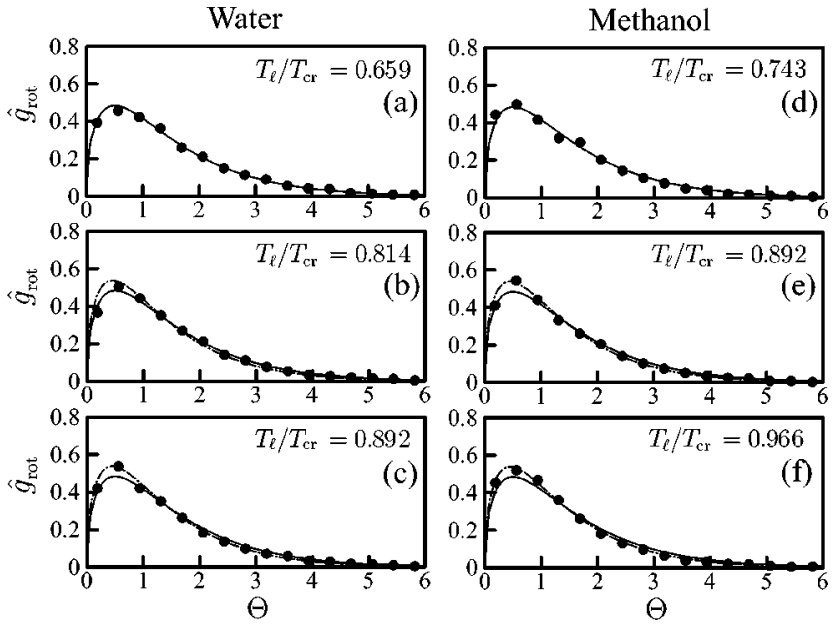

FIG. 13. Distribution function of internal energy of water and methanol molecules evaporating into virtual vacuum at $z^{*}=L_{g}^{*}=4$. The solid curve indicates a one-dimensional normalized Maxwellian $2 \sqrt{\Theta / \pi} \exp (-\Theta)$ and the dash-dotted curves are a Maxwellian with internal temperatures evaluated by MD simulation, $2 \sqrt{\Theta T_{\ell} /\left(T_{2} \pi\right)} \exp \left(-\Theta T_{\ell} / T_{2}\right)$ with $T_{2}=0.9 T_{\ell}\left(\Xi_{p}\right.$ $=0.2 L_{x} L_{y} \delta$ and $\left.\Xi_{e}=0.375 k T_{\ell}\right)$. tributions, while for lower $T_{\ell}$ cases, the rotational energy distributions agree with the Maxwellian $2 \sqrt{\Theta} / \pi \exp (-\Theta)$. As a result, in the case of relatively low $T_{\ell}$, we have

$$
\hat{g}_{\text {rot }}=\hat{g}^{*}
$$

where $\hat{g}^{*}$ is the normalized equilibrium distribution of rotational energy defined by Eq. (5). Note that the distribution of internal energy is related to that of angular velocity through Eq. (16).

Since the correlation coefficient between each component of translational velocity and rotational energy can be proved to be small compared with unity, the distribution function of molecules evaporating into virtual vacuum $f_{\text {evap }}^{\text {sp }}$ may be written as the product of $\rho_{c}, \hat{f}_{\text {trans }}$, and $\hat{g}_{\text {rot }}$,

$$
f_{\text {evap }}^{\text {sp }}=\rho_{c} \hat{f}_{\text {trans }} \hat{g}_{\text {rot }}=2 \rho_{c} \hat{f}^{*} \hat{g}^{*}
$$

where $\rho_{c}$ is defined by Eq. (8). Substituting Eq. (12) into Eq. (26), we obtain

$$
f_{\text {evap }}^{\mathrm{sp}}=\alpha_{e} \rho_{v} \hat{f}^{*} \hat{g}^{*}=\alpha_{e} f^{\mathrm{e}} .
$$

The evaporation part in Eq. (1) is thus validated physically for water and methanol in relatively low temperature case.

In Tables III and IV, $\rho_{c}$ evaluated directly by MD simulations and $(1 / 2) \alpha_{e} \rho_{v}$ are tabulated. It can be seen from the tables that, as $T_{\ell}$ decreases, the numerical value of $\rho_{c}$ and $(1 / 2) \alpha_{e} \rho_{v}$ approach each other, because the translational velocity distribution function approaches the half-Maxwellian.
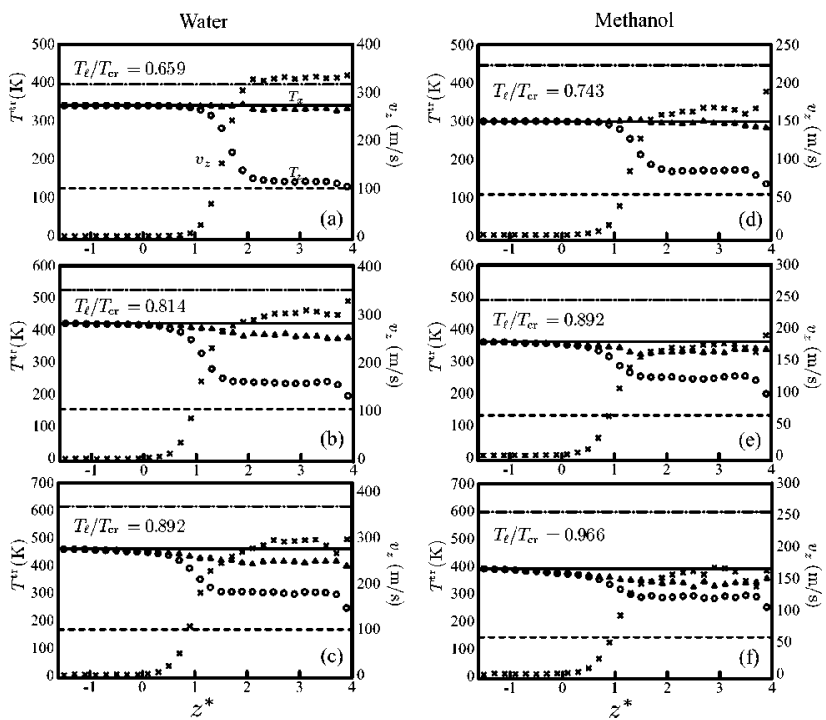

FIG. 14. Translational velocity and temperature of vapor evaporating into virtual vacuum. Symbols are the results of virtual vacuum simulations. The solid line represents $T_{x}=T_{\ell}$, the dash-dotted lines $v z$ $(=316.1,351.3,367.7 \mathrm{~m} / \mathrm{s})$ for water and $v_{z}(=222.6,243.9,253.9 \mathrm{~m} / \mathrm{s})$ for methanol given by Eq. (31). The broken lines are $T z$ $(=123.5,152.6,167.2 \mathrm{~K})$ for water and $T_{z}(=109.0,130.8,141.7 \mathrm{~K})$ for methanol given by Eq. (32) $\left(\Xi_{p}=0.2 L_{x} L_{y} \delta\right)$. 

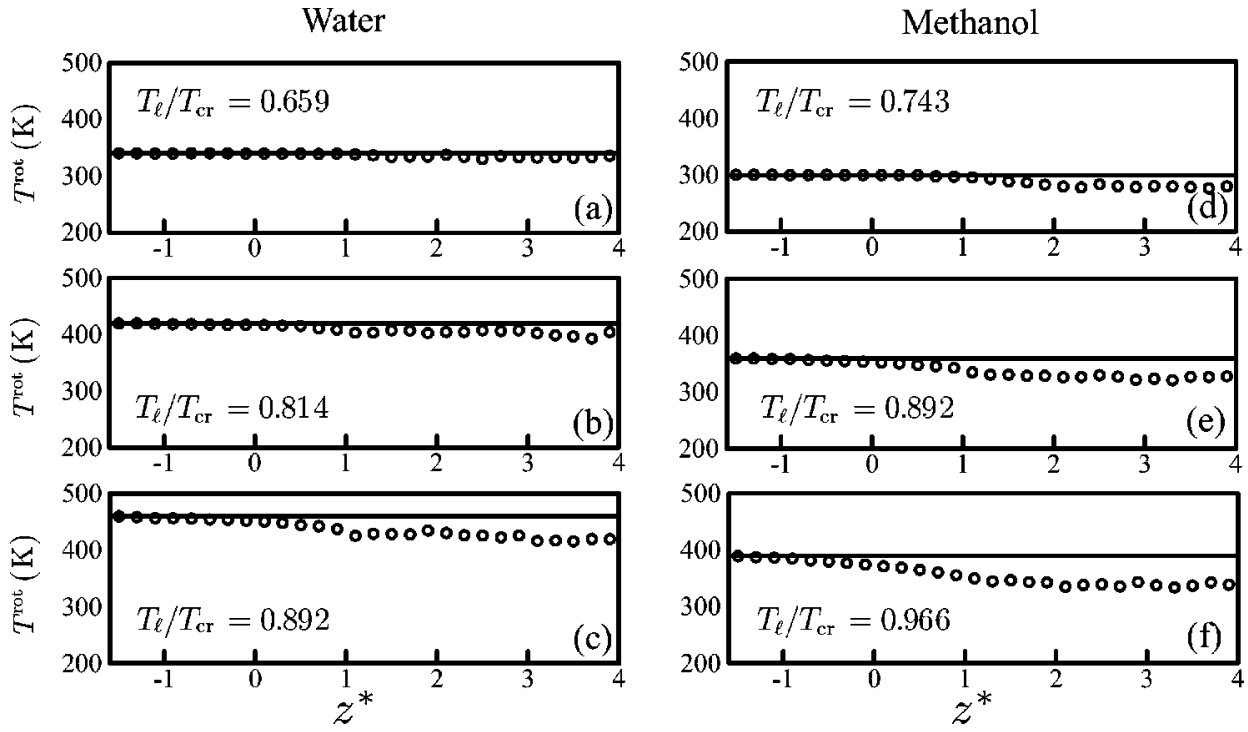

FIG. 15. Rotational temperature of vapor evaporating into virtual vacuum. Symbols are the results of virtual vacuum simulations. The solid line represents $T^{\mathrm{rot}}=T_{\ell}\left(\Xi_{p}=0.2 L_{x} L_{y} \delta\right)$.

\section{E. Velocity and temperature near the interface}

In the kinetic theory, the velocity and temperatures are given by

$$
\begin{aligned}
& v_{j}=\frac{1}{\rho} \int \xi_{j} f d \boldsymbol{\xi} d E \quad(j=x, y, z), \\
& T_{j}^{\mathrm{tr}}=\frac{1}{\rho R} \int\left(\xi_{j}-v_{j}\right)^{2} f d \boldsymbol{\xi} d E \quad(j=x, y, z), \\
& T^{\mathrm{rot}}=\frac{2}{n m \rho R} \int E f d \boldsymbol{\xi} d E,
\end{aligned}
$$

where $T_{j}^{\mathrm{tr}}$ and $T^{\mathrm{rot}}$ are the temperatures associated with the $j$ component of translational velocity and with the rotational energy, respectively. If the distribution $f$ is equal to the halfMaxwellian $\alpha_{e} f^{\mathrm{e}}\left(\xi_{z}>0\right)$, we immediately have

$$
\begin{aligned}
& v_{x}=v_{y}=0, \quad v_{z}=\sqrt{\frac{2 R T_{\ell}}{\pi}}, \\
& T_{x}^{\mathrm{tr}}=T_{y}^{\mathrm{tr}}=T^{\mathrm{rot}}=T_{\ell}, \quad T_{z}^{\mathrm{tr}}=\left(1-\frac{2}{\pi}\right) T_{\ell} .
\end{aligned}
$$

In this section, we shall compare the velocity and temperatures obtained by MD simulation with those given by Eqs. (31) and (32), and make brief comments on the difference between them. The definitions of $v_{j}, T_{j}^{\mathrm{tr}}$, and $T^{\mathrm{rot}}$ in MD simulation are omitted to avoid the repetition of complicated expressions.

As shown in Figs. 14(a) and 14(d), $T_{x}^{\mathrm{tr}}$ (triangle) obtained by MD simulation shows good agreement with the theoretical value $T_{\ell}$ in the vapor region for low $T_{\ell}$ cases. As $T_{\ell}$ increases, however, $T_{x}^{\mathrm{tr}}$ in the vapor region becomes a little bit smaller than the theoretical one. One can also see that the differences of $v_{z}$ (cross) and $T_{z}^{\mathrm{tr}}$ (open circle) by MD simulation from the theoretical ones (dashed line and dash-dotted line) in the vapor region are small at low $T_{\ell}$ and grow with the increase of $T_{\ell}$. This is because the increase of $T_{\ell}$ enhances the appearance of molecules with negative molecular velocity component $\xi_{z}<0$ (see Fig. 11), and the qualitatively similar results have already been found in the case of argon. ${ }^{6}$ Note that, at the absorbing boundary, it happens that a molecule interacting with other one is eliminated, and this leads to the nonmonotonic behavior of velocity and temperature shown in Fig. 14.

Figure 15 shows the rotational temperature distribution near the interface. In the case of lower $T_{\ell}, T^{\text {rot }}$ in the vapor phase almost agrees with the theoretical one, whereas, for higher $T_{\ell}$ case, $T^{\text {rot }}$ decreases (see Fig. 13).

The decrease of $T_{x}^{\mathrm{tr}}$ and $T^{\mathrm{rot}}$ and increase of $T_{z}^{\mathrm{tr}}$ from the corresponding theoretical ones are caused by the local equilibration as a result of intermolecular collisions in the transition layer as in the case of argon. ${ }^{6}$

\section{F. Molecular orientational distribution in virtual vacuum simulation}

In Refs. 15 and 29, an orientational structure near the interface in equilibrium states was studied for water and

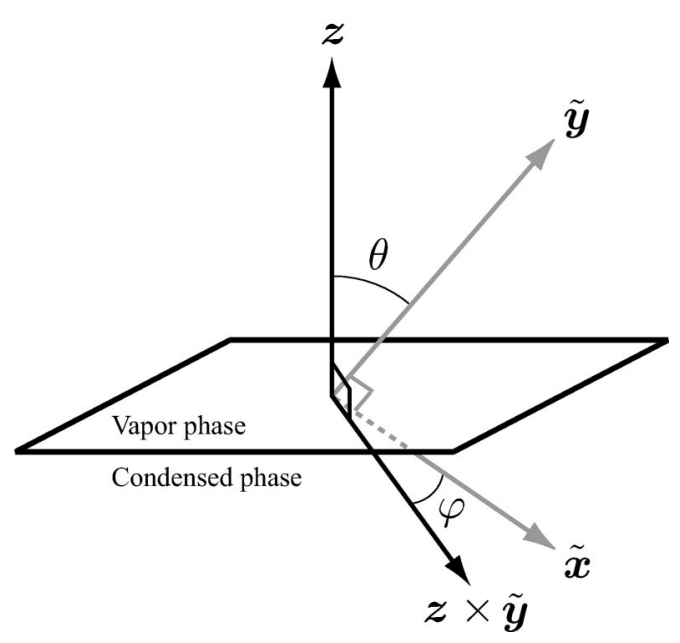

FIG. 16. Definition of orientational angle. The ranges of the variables are, respectively, $0 \leqslant \theta \leqslant \pi / 2$ and $0 \leqslant \varphi \leqslant \pi$ for water, and $0 \leqslant \theta \leqslant \pi$ and $0 \leqslant \varphi$ $\leqslant \pi$ for methanol. 

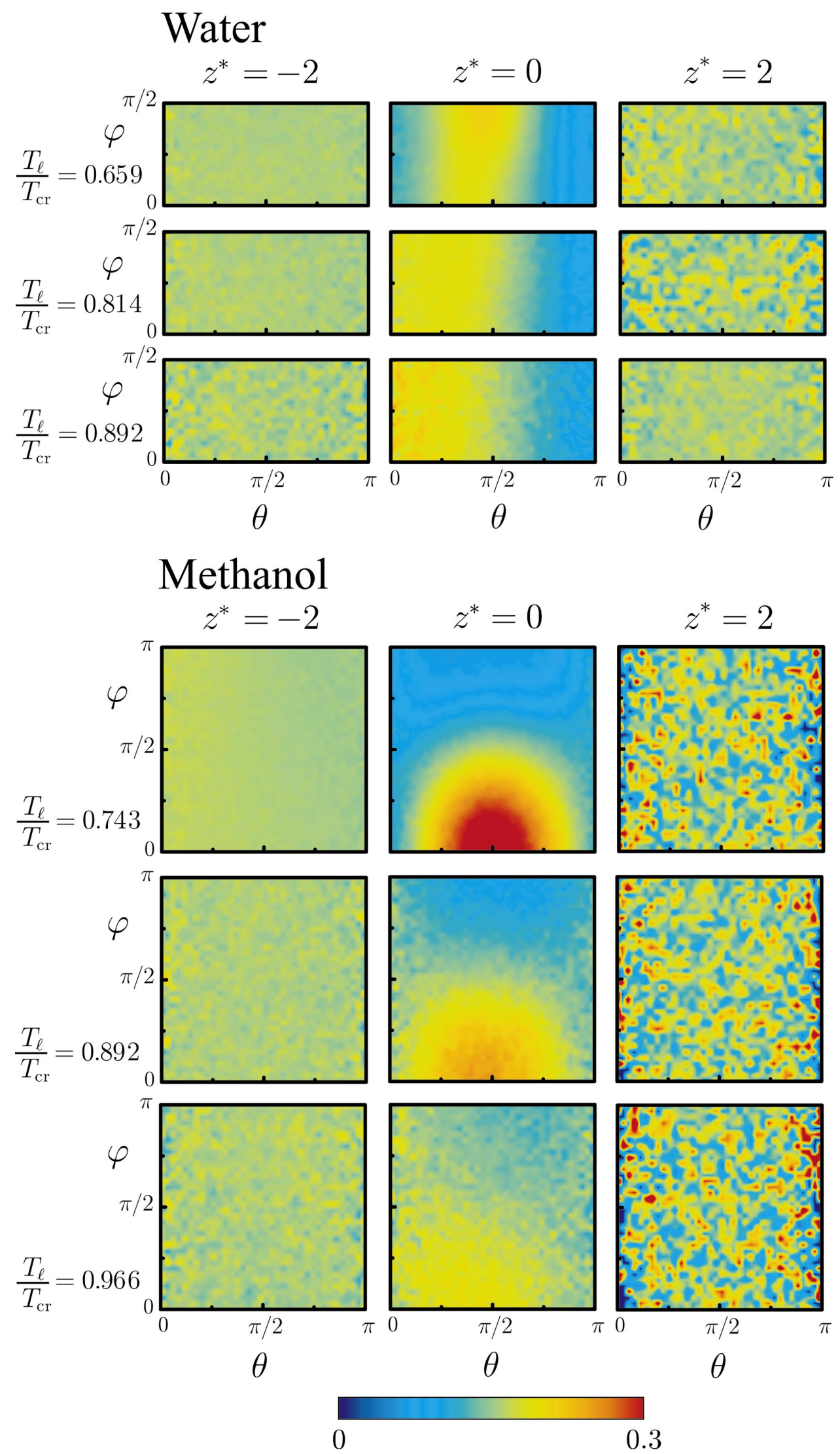

FIG. 17. (Color). Normalized orientational distribution $P(\theta, \varphi)$ in virtual vacuum simulation for water (upper side) and methanol (lower side). The region of $P=0.159$ (green color) denotes isotropic distribution $\left(\Xi_{p}=0.2 L_{x} L_{y} \delta, \Xi_{\theta}=\pi / 36\right.$, and $\left.\Xi_{\varphi}=\pi / 36\right)$. 
methanol by MD simulations, and it was reported that methanol molecules in the interface have a tendency to project their methyl groups to the vapor phase. ${ }^{15,29}$ We shall examine the molecular orientational distribution in the virtual vacuum evaporation states. According to Refs. 15 and 29 , we shall briefly illustrate the definition of angles of orientation of water and methanol molecules. Let $\tilde{\boldsymbol{x}}, \tilde{\boldsymbol{y}}$, and $\widetilde{\boldsymbol{z}}$ be vectors in the directions of principal axes of inertia, which are defined in Fig. 3. As shown in Fig. 16, let $\theta$ be an angle between $\tilde{\boldsymbol{y}}$ and $z$, where $z$ is a vector normal to the interface in the direction from the condensed to vapor phases. Let $\varphi$ be an angle between $\widetilde{\boldsymbol{x}}$ and $\boldsymbol{z} \times \boldsymbol{y}$. Note that, owing to the geometrical symmetry, the range of $\varphi$ is restricted to $[0, \pi / 2]$ for water. A normalized molecular orientational distribution $P(\theta, \varphi)$ can then be calculated by

$$
P(\theta, \varphi)=\frac{1}{\rho N_{s} \Xi_{p} \Xi_{\theta} \Xi_{\varphi} \sin \theta} \sum_{N_{s}} \sum_{i \in\left(\Xi_{p} \cap \Xi_{\theta} \cap \Xi_{\varphi}\right)} m^{i},
$$

where $\Xi_{\theta}$ and $\Xi_{\varphi}$ are one-dimensional volume elements in the one-dimensional $\theta$ and $\varphi$ spaces, respectively. Figure 17 shows the normalized orientational distributions of water and methanol molecules in the steady evaporation state at $z^{*}=-2$ (the inside of the condensed phase), $z^{*}=0$ (the center of the transition layer), and $z^{*}=2$ (the inside of the vapor phase) for several temperatures.

As can readily be seen, at the center of the transition layer, some structure of orientational distribution exists. For water, when $T_{\ell} / T_{\mathrm{cr}}=0.659$ one can see a weak anisotropy near $\theta \approx \pi / 2$ and $\varphi \approx \pi / 2$, and it moves to $\theta \approx 0$ with increase in $T_{\ell}$. For methanol, the strong anisotropy is observed when $T_{\ell} / T_{\mathrm{cr}}=0.743$ and the peak of $P(\theta, \varphi)$ exists around $\theta \approx \pi / 2$ and $\varphi \approx 0$. As $T_{\ell}$ increases, however, the distribution becomes flat.

On the other hand, the distributions inside the condensed phase are isotropic at all temperatures, and those inside the vapor phase can also be regarded as isotropic ones except for fluctuations due to the shortage of samples. That is, although the water and methanol molecules inside the transition layer exhibit the strong orientational ordering, the molecules emitted from the interface does not have any preferential orientations, as expected from the fact that the distribution of angular velocity is almost the Maxwellian.

\section{CONCLUSIONS}

We have carried out the MD simulations of vapor-liquid equilibrium and steady evaporation into the virtual vacuum for water and methanol. The distribution function of molecules evaporating into virtual vacuum has been accurately obtained. We have demonstrated that in relatively low temperature case the distribution function is the product of the evaporation coefficient, the half-Maxwellian of translational molecular velocity, and the equilibrium distribution of rotational energy. The evaporation coefficients of water and methanol are also determined without any ambiguity as a decreasing function of the bulk liquid temperature, and their values are found to become close to unity with decrease in the temperature.
Irrespective of the cluster formation and the preferential orientation of molecules at the interface, which are characteristic features of polyatomic molecule with uneven sharing of the bonding electron pair, we have obtained qualitatively the same results as in the case of argon. As the result of the present study, the physical appropriateness of the evaporation part in the kinetic boundary condition is confirmed for polyatomic molecule as well as for monatomic molecule.

${ }^{1}$ C. Cercignani, Rarefied Gas Dynamics (Cambridge University Press, New York, 2000).

${ }^{2}$ Y. Sone, Kinetic Theory and Fluid Dynamics (Birkhäuser, Boston, 2002). ${ }^{3}$ T. Tsuruta, H. Tanaka, and T. Masuoka, "Condensation/evaporation coefficient and velocity distributions at liquid-vapor interface," Int. J. Heat Mass Transfer 42, 4107 (1999).

${ }^{4}$ R. Meland and T. Ytrehus, "Boundary condition at a gas-liquid interface," in Rarefied Gas Dynamics: 22nd International Symposium, edited by T. J. Bartel and M. A. Gallis (American Institute of Physics, New York, 2001), p. 583.

${ }^{5}$ A. Frezzotti and L. Gibelli, "A kinetic model for equilibrium and nonequilibrium structure of the vapor-liquid interface," in Rarefied Gas Dynamics: 23rd International Symposium, edited by A. D. Ketsdever and E. P. Muntz (American Institute of Physics, New York, 2003), p. 980.

${ }^{6}$ T. Ishiyama, T. Yano, and S. Fujikawa, "Molecular dynamics study of kinetic boundary condition at an interface between argon vapor and its condensed phase," Phys. Fluids 16, 2899 (2004).

${ }^{7}$ W. B. Takens, W. Mischke, J. Korving, and J. J. M. Beenakker, "A spectroscopic study of free evaporation of sodium," in Rarefied Gas Dynamics, edited by H. Oguchi (University of Tokyo Press, Tokyo, 1984), p. 976.

${ }^{8}$ C. Cercignani, W. Fiszdon, and A. Frezzotti, "The paradox of the inverted temperature profiles between an evaporating and a condensing surface," Phys. Fluids 28, 3237 (1985).

${ }^{9}$ The collision mass flux $\left\langle J_{\text {coll }}\right\rangle$ is defined as

$$
\left\langle J_{\text {coll }}\right\rangle=-\iiint_{\xi_{z}<0} \int_{0}^{\infty} \xi_{z} f_{\text {coll }} d E d \xi_{x} d \xi_{y} d \xi_{z},
$$

where $f_{\text {coll }}$ is the distribution function for molecules incoming to the interface, and $J_{\text {cnds }}$ is evaluated from Eq. (14) and $\left\langle J_{\text {ref }}\right\rangle=\left\langle J_{\text {out }}\right\rangle-\left\langle J_{\text {evap }}^{\text {sp }}\right\rangle$ (see also Fig. 1).

${ }^{10} \mathrm{G}$. Nagayama and T. Tsuruta, "A general expression for the condensation coefficient based on transition state theory and molecular dynamics simulation," J. Chem. Phys. 118, 1392 (2003).

${ }^{11}$ M. P. Allen and D. J. Tildesley, Computer Simulation of Liquids (Clarendon, Oxford, 1987).

${ }^{12}$ W. L. Jorgensen, J. Chandrasekhar, J. D. Madura, R. W. Impey, and M. L. Klein, "Comparison of simple potential functions for simulating liquid water," J. Phys. Chem. 79, 926 (1983).

${ }^{13} \mathrm{~W}$. L. Jorgensen, "Optimized intermolecular potential functions for liquid alcohols," J. Phys. Chem. 90, 1276 (1986).

${ }^{14}$ J. T. Wescott, L. R. Fisher, and S. Hanna, "Use of thermodynamic integration to calculate the hydration free energies of n-alkanes," J. Chem. Phys. 116, 2361 (2002).

${ }^{15}$ M. Matsumoto and Y. Kataoka, "Study on liquid-vapor interface of water. I. Simulational results of thermodynamic properties and orientational structure," J. Chem. Phys. 88, 3233 (1988).

${ }^{16}$ M. Kettler, I. Nezbeda, A. A. Chialve, and P. T. Cummings, "Effect of the range of interactions on the properties of fluids. Phase equilibria in pure carbon dioxide, acetone, methanol, and water," J. Phys. Chem. B 106, 7537 (2002).

${ }^{17}$ M. Mecke, and J. Winkelmann, "Molecular dynamics simulation of the liquid-vapor interface of dipolar fluids under different electrostatic boundary conditions," J. Chem. Phys. 114, 5842 (2001).

${ }^{18}$ M. Hloucha, A. K. Sum, and S. I. Sandler, "Computer simulation of acetonitrile and methanol with $a b$ initio-based pair potentials," J. Chem. Phys. 113, 5401 (2000).

${ }^{19}$ L. Vega, E. de Miguel, and L. F. Rull, "Phase equilibria and critical behavior of square-well fluids of variable width by Gibbs ensemble Monte Carlo simulation," J. Chem. Phys. 96, 3233 (1992).

${ }^{20} \mathrm{~W}$. Wagner and A. Pruss, "The IAPWS formulation 1995 for the thermodynamic properties of ordinary water substance for general and scientific 
use," J. Phys. Chem. Ref. Data 31, 387 (2002).

${ }^{21}$ R. D. Goodwin, "Methanol thermodynamic properties from 176 to $673 \mathrm{~K}$ at pressures to 700 bar," J. Phys. Chem. Ref. Data 16, 799 (1987).

${ }^{22}$ S. I. Anisimov, D. O. Dunikov, S. P. Malyshenko, and V. V. Zhakhovskii, "Properties of a liquid-gas interface at high-rate evaporation," J. Chem. Phys. 110, 8722 (1999).

${ }^{23}$ In high temperature case, the transition layer extends close to $z^{*} \cong 2$, and hence we cannot set $L_{\rho}^{*}$ at $z^{*} \leqslant 2$.

${ }^{24}$ T. L. Hill, Statistical Mechanics: Principles and Selected Applications (McGraw-Hill, New York, 1956).

${ }^{25}$ M. Matsumoto, "Molecular dynamics of fluid phase change," Fluid Phase Equilib. 144, 307 (1998).
${ }^{26}$ R. Marek and J. Straub, "Analysis of the evaporation coefficient and the condensation coefficient of water," Int. J. Heat Mass Transfer 44, 39 (2001).

${ }^{27}$ S. Fujikawa, T. Yano, K. Kobayashi, K. Iwanami, and M. Ichijo, "Molecular gas dynamics applied to phase change processes at a vapor-liquid interface: Shock-tube experiment and MGD computation for methanol," Exp. Fluids 37, 80 (2004).

${ }^{28}$ R. C. Reid, J. M. Prausnitz, and T. K. Sherwood, The Properties of Gases and Liquids (McGraw-Hill, New York, 1977).

${ }^{29} \mathrm{M}$. Matsumoto and Y. Kataoka, "Molecular orientation near liquid-vapor interface of methanol: Simulational study," J. Chem. Phys. 90, 2398 (1989). 